\title{
La invención de problemas y sus ámbitos de investigación en educación matemática
}

\author{
Johan Espinoza G. \\ jespinoza@una.cr \\ Universidad Nacional \\ Costa Rica
}

\author{
Jose Luis Lupiañez G. \\ lupi@ugr.es \\ Universidad de Granada \\ España
}

\author{
Isidoro Segovia A. \\ isegovia@ugr.es \\ Universidad de Granada \\ España
}

Recibido: Octubre 9, $2013 \quad$ Aceptado: Febrero 6, 2013

\begin{abstract}
Resumen. Nos centramos en estudiar las investigaciones relacionadas con la invención de problemas matemáticos para categorizarlas en ámbitos de investigación y educación. Así, se pretende aportar información relevante a investigadores y educadores en Educación Matemática, sobre los diferentes intereses de investigación de este campo y mostrar la riqueza que aporta este tipo de actividades en clases. Los resultados muestran que ha sido estudiada para desarrollar el pensamiento matemático de los estudiantes, en la mejora de las actitudes hacia la matemática, como característica de la actividad creativa o talento excepcional, en la identificación de estudiantes con talento en matemática, en los procesos de resolución de problemas, para observar la comprensión matemática de los estudiantes, como herramienta de evaluación, entre otros.
\end{abstract}

Palabras clave: Problemas matemáticos, invención de problemas, resolución de problemas.

\begin{abstract}
We focus on research study related to mathematical problem posing for categorizing in research and education. It seeks to provide relevant information to researchers and educators in Mathematics on different research interests in this area, and to display how class activities are enriched . Results show that it has been studied to develop student mathematical thinking, improve attitudes towards mathematics, as a trait of creative activity or exceptional talent, in identifying students talented in mathematics, in the processes of problem solving, to observe mathematical understanding of students, as a tool for evaluation, among others.
\end{abstract}

KeyWords: Mathematical problem, problem posing, problem solving 


\subsection{Introducción}

Desde la década de los ochenta del siglo pasado, la resolución de problemas es reconocida como una actividad central de la matemática escolar (NCTM, 1980, 1989). De hecho, algunos autores destacan que en todas las clases de matemática de cualquier país, se puede observar a los estudiantes resolver problemas matemáticos (Silver, 1994). A partir de esa etapa surgen diversas líneas de investigación centradas en la resolución de problemas, entre las cuales, la invención de problemas matemáticos es una de ellas (Castro, 2008).

Uno de los primeros investigadores que reconoció la importancia de tal actividad fue Krutetskii (1976), en su estudio sobre la comprensión de la naturaleza de las habilidades matemáticas de niños considerados con talento matemático. También Polya (1979), en su propuesta sobre el proceso de resolución de un problema, hace referencia a esta actividad, al cuestionarse si el problema puede ser planteado de manera diferente o variar el problema descartando parte de la solución.

El interés sobre la invención de problemas también se ve reflejado en reportes curriculares como los "Estándares sobre el currículo y evaluación para las matemáticas escolares" (NCTM, 1989); en proyectos de evaluación internacionales como PISA 2012 (OECD, 2013); y en propuestas que establecen perfiles profesionales para la enseñanza de la matemática (NCTM, 1991 y 2000), que sugieren un incremento en el uso de dicha actividad en las clases de matemáticas.

De igual forma, algunos investigadores en educación matemática (Freudenthal, 1973; Ellerton 1986; Brown y Walter, 1990; Sullivan, Clarke y Clarke, 2012), reconocen este tipo de actividad como fundamental dentro de la experiencia matemática de los estudiantes y mencionan el gran valor educativo, que tiene a lo largo del tiempo, que los estudiantes de todos los niveles inventen problemas en clase.

Parece relevante, por tanto, categorizar las diferentes investigaciones que se han realizado hasta ahora y presentarlas como ámbitos de investigación y de educación en invención de problemas matemáticos. Así, el objetivo de este estudio es aportar información relevante a docentes e investigadores en Educación Matemática, sobre cómo se ha investigado la invención de problemas matemáticos y cuáles son las virtudes de emplear este tipo de actividades en clases. De igual forma, se pretende mostrar la riqueza que presenta este tipo de actividades en el desarrollo del pensamiento matemático de los estudiantes, como actividad matemática dentro del salón de clase, y como campo de investigación en Educación Matemática.

Sin embargo, antes de presentar cómo se ha investigado dicho tema, realizamos un breve preámbulo sobre las diferentes concepciones que presenta la invención de problemas matemáticos.

\subsection{La invención de problemas en la educación matemática}

El término invención de problemas, también conocido en la literatura en inglés como "problem posing" (Brown y Walter, 1993; Kilpatrick, 1987; Silver, 1994; English, 1997), implica la formulación de nuevos 
problemas y la reformulación de situaciones dadas (Silver, 1994; English, 1997; Silver y Cai, 1996).

En este sentido, los estudiantes pueden inventar problemas durante la solución de un problema complejo (Silver, Mamona-Down, Leung y Kenny, 1996), cuando realizan cambios al mismo. Así, podrían reformularlo y/o personalizarlo (Silver, 1994) disminuyendo el tamaño de los números empleados o estudiar un caso particular de la situación dada, con el objetivo de comprender mejor el problema y así buscar una solución al mismo. Por ejemplo, en el trabajo de Polya (1979), aparece esta componente esencial de la actividad matemática, cuando se cuestiona ¿cómo podemos plantear el problema de manera diferente?, ¿cómo variar el problema descartando parte de la condición?

Este proceso también puede ocurrir antes de resolver un problema; cuando lo que se persigue no es la solución del mismo, sino formular problemas matemáticos a partir de una situación o experiencia dada previamente al estudiante (Silver, 1994). Por ejemplo, en el estudio de Cázares (2000), se presentó a estudiantes de primaria varias tarjetas con diferentes ilustraciones de situaciones relacionadas con su contexto, de las cuales debían escoger algunas y plantear varios problemas matemáticos. De igual forma, en la investigación de Espinoza (2011), los estudiantes debían inventar un problema aritmético que consideraran difícil de resolver, a partir de dos situaciones, una de ellas expresada mediante una ilustración y otra con base en una situación expuesta de forma escrita.

Por último, este tipo de actividades se pueden realizar posterior al proceso de resolución de un problema, cuando se le indica a los estudiantes que modifiquen el objetivo, meta, condición o pregunta del mismo, con el fin de generar nuevos enunciados (Silver, 1994). Esta concepción es utilizada por Brown y Walter (1993), en su estrategia denominada “¿What if not?", la cual consiste en cambiar las condiciones y restricciones del problema, para así plantear nuevos e interesantes situaciones que pueden llevar a resultados relevantes.

Por otra parte, se identifican tres formas en las cuales se podrían formular problemas: situación libre, situaciones semiestructuradas y situaciones estructuradas (Stoyanova, 1998). En la primera, los estudiantes no tienen restricciones para inventar problemas; mientras que en las situaciones semiestructuradas se les propone que planteen enunciados con base en alguna experiencia o en contextos expresados mediante ilustraciones o de forma textual. Por último, las situaciones estructuradas, son aquellas en las que se reformulan los problemas dados o se cambia la condición del mismo.

De esta forma, los estudiantes pueden hacer sus producciones a partir de una ilustración que presente o no información numérica, que se resuelvan con base en operaciones aritméticas dadas, mediante alguna información presentada de forma textual, modificando la respuesta de un enunciado o mediante el planteamiento libre de una situación.

Así, con base en la literatura consultada, al hecho de inventar problemas se le ha dado distintas denominaciones por diferentes autores. Se le ha designado como generación de problemas o reformulación de problemas dados (Silver, 1994), formulación de problemas (Kilpatrick, 1987) y planteamiento de problemas (Brown y Walter, 1990). A nuestro parecer, estas denominaciones hacen referencia al mismo hecho, por lo que utilizaremos con más frecuencia la expresión invención de problemas.

Una vez expuestas las ideas fundamentales relacionadas con este tipo de actividades, procedemos a presentar algunos ámbitos de investigación en invención de problemas matemáticos. 


\section{3 Ámbitos de investigación en invención de problemas matemáticos}

Diferentes investigadores en Educación Matemática han estudiado la invención de problemas matemáticos con distintos propósitos. A continuación presentamos de forma resumida las investigaciones encontradas en una búsqueda sobre el tema, clasificándolas según el propósito para el cual fueron empleadas.

\section{La invención de problemas como característica de la actividad creativa o talento excepcional}

Algunos estudios ponen de manifiesto que la formulación de problemas tiene una incidencia positiva en el desarrollo de la creatividad de los alumnos. Por ejemplo Silver (1994), estudió la creatividad en un grupo de estudiantes y valoró la fluidez de acuerdo con el número de problemas planteados, la flexibilidad con respecto a la diversidad de problemas propuestos y la originalidad de acuerdo con la cantidad de soluciones propuestas.

Este tipo de actividades también han sido empleadas para explorar el desempeño de estudiantes con altas capacidades en matemáticas. Esto queda de manifiesto en el estudio de Krutetskii (1976), quien utilizó como una de las medidas de talento excepcional una tarea de invención de problemas para observar los procesos cognitivos de 192 niños entre los 6 y 16 años. La investigación concluye que existe una relación positiva entre la habilidad para proponer problemas, el grado de creatividad y el talento matemático. Además, identificó en sus producciones la tendencia a preferir formas de pensamiento visuales-espaciales o una forma lógica-analítica.

De igual forma en el trabajo de Ellerton (1986), se emplearon actividades de planteamiento de problemas para comparar los problemas matemáticos planteados por dos grupos de niños, uno con mayor habilidad matemática que otro. Los resultados de este estudio muestran diferencias importantes entre ambos grupos. Por ejemplo, se observó que las producciones de los estudiantes con más habilidad requieren mayor dificultad de cálculo, presentan una mayor cantidad de operaciones, implican un sistema numérico más complejo y utilizan el lenguaje matemático con mayor fluidez que sus compañeros menos capaces. También concluye que los estudiantes más hábiles mostraron un mayor dominio de la tarea propuesta, ya que sus enunciados presentaron más coherencia y consistencia que los inventados por los estudiantes menos hábiles.

Más recientemente Kesan, Kaya y Güvercin (2010), estudiaron el efecto de las actividades de invención de problemas en el desarrollo de habilidades matemáticas de 40 estudiantes con talento matemático, con edades entre 14 y 15 años, de un colegio llamado "School for Kazakh gifted students". Estos estudiantes fueron divididos en dos grupos, de forma que uno de ellos participó en actividades de invención de problemas (grupo experimental) y el otro grupo recibió una instrucción tradicional (grupo control).

Antes del proceso de instrucción de invención de problemas, ambos grupos realizaron como pre-test, el test de Habilidad en Resolución de Problemas Matemáticos, identificado por sus siglas en inglés como MPSAT . Luego se aplicó nuevamente como pos-test, con el propósito de medir el efecto de la instrucción de invención de problemas, sobre el desarrollo de las habilidades matemáticas de estudiantes con talento matemático. 
Los resultados muestran que antes de la instrucción no hay diferencias significativas entre las medias de ambos grupos (control y experimental); sin embargo, sí se encontraron diferencias después la instrucción de invención de problemas en el pos-test. Los investigadores concluyen que hay diferencias significativas entre las notas medias del test MPSAT para los estudiantes del grupo experimental, resultando así que las actividades de invención de problemas son efectivas en su rendimiento, especialmente para ejercicios no rutinarios.

Por último, en el estudio de Espinoza (2011), se construyeron dos tareas de invención de problemas semiestructuradas, las cuales se aplicaron a dos grupos de 20 estudiantes. El primero, denominado grupo talento, conformado por estudiantes que participaron en el proyecto ESTALMAT-Andalucía ${ }^{1}$, España y el otro denominado grupo estándar, formado por estudiantes de un colegio público normal de Salobreña, España.

Los resultados de esta investigación muestran que los problemas planteados por estudiantes con talento presentan una mayor riqueza en cuanto a la longitud del enunciado, tipo de proposición interrogativa, tipo de número, cantidad de procesos y pasos implicados en la solución del problema que sus compañeros del grupo estándar. Además, se encontraron diferencias en el tipo de estructura semántica y en la cantidad de relaciones semánticas implicadas en la solución del problema.

Estas diferencias, según la investigación, se reflejaron en la resolución de los problemas planteados por este grupo, ya que dan la sensación de mayor dificultad, puesto que al leer el enunciado no se identifica de forma inmediata un procedimiento para resolverlos.

\section{La invención de problemas como característica de una enseñanza orientada a la responsabilidad en el aprendizaje}

Algunos investigadores han realizado estudios que muestran que las actividades de invención de problemas producen efectos positivos en el proceso de aprendizaje de los estudiantes. Al respecto, Brown y Walter $(1990,1993)$, han explorado la invención de problemas con este propósito. Los resultados de sus experiencias muestran, que si los estudiantes tienen la oportunidad de crear y explorar sus propios problemas, entonces éstos tomarán más responsabilidad en su propio aprendizaje.

También se ha observado que este tipo de actividades dan lugar a un nivel muy visible de compromiso, curiosidad y entusiasmo durante la clase, así como una mayor responsabilidad de parte del estudiante en la construcción de su conocimiento (Cunningham, 2004). Esto también se refleja en algunos estudios (Cázares, 2000; Espinoza, 2011), donde se le indica a los estudiantes que planteen problemas que, a su parecer, sean difíciles de resolver, ya que esto los motiva a comprometerse con la tarea encomendada.

Brueckner (1932; citado en Cázares, 2000), también diseñó un actividad de formulación de problemas con el propósito de ayudar a sus estudiantes a desarrollar el sentido de las relaciones numéricas y la generalización de los conceptos del número y observó que los estudiantes se involucraron en los procedimientos para expresar en términos matemáticos las distintas situaciones que se les presentaron.

\footnotetext{
${ }^{1}$ El proyecto ESTALMAT-Andalucía se centra en la estimulación, durante dos cursos académicos, del talento matemático precoz de 25 alumnos de centros educativos de la región de Andalucia, España, de 12 o 13 años, los cuales son seleccionados mediante la aplicación de pruebas-actividades propuestas por el equipo de profesores para una evaluación externa. http://thales.cica. es/estalmat/
} 
No obstante, es posible que este tipo de actividades requieran más tiempo para alcanzar los objetivos propuestos, pero quizás con mayores beneficios en el proceso de enseñanza aprendizaje de los estudiantes. Al respecto Brown y Walter (1993) señalan que:

\begin{abstract}
“Podría tomar más tiempo cubrir un conjunto de conocimientos predeterminados y quizá se podría cubrir menos material, pero los estudiantes probablemente tomarán conciencia de su propio estilo de pensar, actitudes hacia el trabajo con otros, y también acerca de la naturaleza y el propósito de la disciplina" (p. 26).
\end{abstract}

\title{
La invención de problemas como una ventana para observar la comprensión matemática de los estudiantes
}

La actividad de inventar problemas también ha sido estudiada como medio que permite conocer las habilidades de los estudiantes, así como la comprensión de los conceptos matemáticos aprendidos. Por ejemplo, en el estudio de Pelczer y Gamboa (2008), se propuso a un grupo de 21 estudiantes que plantearan tres problemas matemáticos y se observó que ellos parten de una idea general o punto de referencia, luego realizan un proceso de generación-evaluación, durante el cual muestran sus habilidades y conocimientos aprendidos previamente.

Ellerton (1986), también estudió las ideas matemáticas expresadas por un grupo de niños de 11 a 13 años en las tareas de invención de problemas y encontró que sus producciones muestran no solo su comprensión y nivel de desarrollo de conceptos, sino también su percepción sobre la naturaleza de las matemáticas y su actitud hacia esta disciplina.

De igual forma, la invención de problemas no sólo da la oportunidad al estudiante de demostrar sus conocimientos y qué es capaz de hacer con ellos, sino que también permite al profesor observar patrones en el aprendizaje y el pensamiento matemático de los estudiantes (Kwek y Lye, 2008).

En España se han realizado algunas investigaciones en esta dirección. Por ejemplo, Luque (2004), se centró en estudiar los conocimientos sobre fracciones y las operaciones que ponen en juego un grupo de estudiantes de tercer curso de secundaria cuando inventan problemas. La comprensión de dichos conceptos se valoró en función del tipo de problema que inventen, el significado de fracción y las representaciones de las fracciones utilizadas en los problemas propuestos.

El autor concluye que si un estudiante es capaz de sumar fracciones, pero no es capaz de identificar una situación que requiera suma de fracciones, entonces no tiene una buena comprensión de tal concepto, por lo que existiría un desequilibrio entre los tipos de conocimiento conceptual y procedimental.

Ayllón (2012) también empleó tareas de invención de problemas para estudiar el significado y los diferentes usos y contextos que un grupo de profesores en formación en educación primaria dan a tres tipos de números: naturales, enteros negativos y racionales. De igual forma, ella pretendía estudiar cómo este tipo de actividades pueden ayudar a desarrollar la comprensión sobre conocimiento de los números en dicha población. De acuerdo con el estudio, las mayores dificultades se presentaron en el conjunto de los números racionales, donde se encontraron una mayor cantidad de enunciados erróneos y en el que más dificultad hubo para identificar los elementos que pertenecen a un problema. 


\section{La invención de problemas como herramienta para evaluar el aprendizaje de conocimientos matemáticos}

En el apartado anterior se evidenció que la generación de problemas permite al profesor tener una visión de la comprensión de los conceptos y procedimientos matemáticos que tienen sus estudiantes, así como profundizar en la construcción de la comprensión de dichos conceptos (English, 1997; Lin, 2004). Ante esto, algunos investigadores y educadores se han interesado en estudiarla y emplearla como una herramienta para evaluar el aprendizaje de conocimientos matemáticos de los estudiantes (Kwek y Lye, 2008; Silver y Cai, 2005).

Al respecto, Silver y Cai (2005), mencionan que si la invención de problemas se utiliza como una actividad más en el proceso de enseñanza de la matemática, entonces también podría ser incorporada de alguna forma dentro de la evaluación de clase, como un medio para comprobar la comprensión o capacidad de los estudiantes. Estos mismos autores mencionan que la invención de problemas podría ser una buena forma para evaluar si el estudiante comprende que cualquier problemática que involucre el concepto de división, puede usarse como modelo de muchas situaciones problema y que la respuesta depende de las características de la situación y de la naturaleza de la cantidad implicada. También se resalta por parte de Lin (2004), que un aspecto positivo que tiene ésta como herramienta de evaluación con respecto a otras, es que no está separada del proceso de instrucción sino inmersa en ella.

Así, los docentes pueden emplear este tipo de actividades para valorar el grado de comprensión de los conceptos matemáticos aprendidos por los estudiantes luego de explicar el contenido a enseñar. De igual forma, podría emplearse como evaluación diagnóstica al iniciar un año lectivo o tema, o simplemente como actividad de clase que permite al estudiante valorar su propio aprendizaje y profundizar en los conceptos aprendidos.

Por último, algunos estudios (Ellerton, 1986; Cázares 2000; Silver y Cai, 2005; Espinoza, 2011; Ayllón, 2012), mencionan elementos a tomar en cuenta para valorar las producciones que resultan de proponer taras de invención de problemas. Se pueden citar el número de problemas generados, el tipo de número empleado, la extensión y complejidad lingüística del enunciado, la creatividad, el tipo de pregunta, la cantidad de procesos implicados en la solución, la complejidad matemática y la dificultad percibida para resolverlo, etc.

\section{La invención de problemas como medio para mejorar la disposición y las actitudes hacia las matemáticas}

Además de la relevancia de la invención de problemas como promotora de la responsabilidad de los escolares en su propio aprendizaje que describimos antes, otros trabajos enfatizan el papel que puede jugar para mejorar la disposición y las actitudes de los estudiantes hacia la matemática (Brown y Walter, 1990, 1993; Silver, 1994; Silver, Mamona-Downs, Leung, Kenney, 1996; English, 1997). Al respecto Winograd (1991, citado en Cázares, 2000), estudió el comportamiento cognitivo de un grupo de estudiantes durante el proceso de inventar problemas y observó que se encontraban altamente motivados para participar en las actividades de matemática, al proponer y compartir con sus compañeros de clase problemas de diferente nivel de dificultad.

Akay y Boz (2010), examinaron el efecto que tuvo el planteamiento de problemas sobre las actitudes hacia la matemática de 82 profesores en formación. En su estudio concluyen que esta técnica es efectiva en la mejora de las actitudes hacia la matemática, reduciendo su ansiedad y motivando incluso a 
aquellos estudiantes que tienen poco conocimiento sobre esta asignatura. Además, observaron que este tipo de actividades crean una atmósfera optimista dentro de la clase, estimulando a los estudiantes a concentrarse más sobre las actividades de pensamiento crítico, alejándose de algunas conductas negativas y mostrando una mayor disposición a asistir a las lecciones de matemática.

\section{La invención de problemas como medio para mejorar la capacidad de resolución de problemas}

Algunos autores (Polya, 1979; Cázares, 2000; English, 1997), ponen de manifiesto la importancia que tienen las actividades de invención de problemas dentro del proceso de resolución. Por ejemplo Osbon (1963; citado en Castro, 1991), menciona que se debe dividir el problema en subproblemas para facilitar la resolución del mismo. De igual forma, Rossman (1971; citado en Castro, 1991), sugiere la necesidad de reformularlo para facilitar la solución del mismo. Por último, en la fase "looking Back" citada por Polya (1979) y en el método IDEAL empleado por Bransford y Stein (1986), aparece dicha componente.

En este sentido Leung y Silver (1997), destacan el valor que tiene la invención de problemas en la mejora de las habilidades de los estudiantes al resolver problemas matemáticos y citan algunas investigaciones (Keil, 1965; Pérez, 1985; Winograd, 1991), que evidencian la influencia positiva que tiene este tipo de actividades en el desarrollo de estrategias de resolución. De igual forma, Cannor y Hawkings (1936, citados en Cázares, 2000), afirman que si los estudiantes generan sus propios problemas, entonces incrementarán su habilidad para aplicar los conceptos y destrezas necesarios para su resolución.

Otros estudios ponen de manifiesto la estrecha relación que existe entre la invención y la resolución de problemas. Por ejemplo, en el trabajo de Walter y Brown (1977) se estudió la interdependencia entre ambas tareas matemáticas y se encontró que el rendimiento en una de ellas tiene importantes repercusiones en la otra, y viceversa. Silver (1997), también abordó la relación entre la invención y resolución de problemas, a partir de las diferencias entre el tipo de problemas planteados por solucionadores exitosos y por los no exitosos.

De igual forma, Silver y Cai (1996), indagaron sobre dicha relación y concluyen que el rendimiento de los estudiantes en la resolución de problemas tuvo una alta correlación con su rendimiento en el planteamiento de problemas. Del mismo modo, en el trabajo de English (1997), se pone de manifiesto que los estudiantes con mayor habilidad en resolución de problemas, plantean problemas con una estructura más compleja que sus compañeros con menos habilidad en resolución de problemas.

Por último, William (2003, citado en Luque, 2004), plantea que la invención de problemas y posterior solución, permite al estudiante descubrir sus limitaciones, comprobar sus propios errores, conocer sus propias estrategias y dificultades, convirtiéndolos en mejores resolutores.

\section{Implicaciones en el proceso de invención de problemas}

Dada la relevancia que tienen las actividades de invención de problemas como actividad matemática y las bondades reconocidas dentro del proceso de enseñanza aprendizaje de esta disciplina, es que algunos autores recomiendan una serie de aspectos a tomar en cuenta, cuando se propone a los estudiantes generar problemas matemáticos.

En relación con la situación presentada a los estudiantes para que inventen problemas, Moses, Bjork y Goldenberg (1990), hacen referencia a tres cuestionamientos: la clase de información que proporciona 
el problema, qué tipo de información permanece desconocida (y requerida) y qué tipo de restricciones están implicadas en la respuesta.

Otro autor que aborda los procedimientos implicados en el proceso de invención de problemas es English (1997), quien afirma que los estudiantes necesitan un marco de conocimientos que les permita hacer frente a las actividades cognitivas que conlleva el inventar problemas. Así, propone que un estudiante debe comprender lo que es un problema, reconocer su estructura e identificar estructuras similares para que pueda inventar un problema. Este mismo autor menciona que se debe considerar cómo conciben los estudiantes los diferentes tipos de problemas, así como los procedimientos rutinarios y no rutinarios para resolverlos. Por último, indica que la habilidad que posea el estudiante para comprender e interpretar las situaciones matemáticas de diversas maneras mejora la invención de problemas.

\subsection{Conclusiones}

En primera instancia, reconocemos la invención de problemas como una actividad central de la experiencia matemática de cualquier estudiante, la cual puede emplearse durante la resolución de un problema matemático, luego de resolver un problema o cuando el sujeto se enfrenta ante una situación conocida previamente, para la cual no hay una formulación matemática. Su importancia queda manifiesta en estudios realizados por relevantes investigadores y educadores en matemática y en reportes curriculares como el NCTM, quienes mencionan el gran valor educativo que tiene este tipo de actividades.

En este sentido, a partir del estudio se evidencia que en este tipo de actividades los estudiantes pueden reconocer mejor las partes de un problema y establecer relaciones. Además, se responsabilizan más en su propio aprendizaje, muestran mayor curiosidad y entusiasmo durante la clase, así como una mejor actitud hacia el trabajo.

De igual forma, los profesores pueden planear sistemáticamente tareas de invención de problemas con varios fines. Primero, pueden examinar y valorar directamente la comprensión de los conceptos matemáticos de los estudiantes, ya que en los problemas generados queda plasmado el significado que tienen de los conocimientos aprendidos, habilidades matemáticas, creatividad, profundización de los conceptos, patrones, uso de los números y cantidades, entre otros.

También podrían emplearse para mejorar las competencias de resolución de problemas que tienen los estudiantes. Al respecto, consideramos que el planteamiento de problemas tiene un rol complementario en los procesos de resolución de problemas, pues agrega una mayor representación matemática al mismo. Esto se da porque en la reformulación de un problema dado los estudiantes descomponen el mismo en más partes y logran comprender mejor en qué consiste el problema.

Además, se puede utilizar como un instrumento para evaluar los aprendizajes alcanzados por los estudiantes, ya sea de forma diagnóstica o al finalizar un tema, o como una herramienta que permite estudiar e identificar el talento matemático. Sin embargo, existen pocos estudios que aborden herramientas que permitan valorar las producciones derivadas de una tarea de invención de problemas. 
$\mathrm{Al}$ respecto, se pueden mencionar los esquemas utilizados en los trabajos de Espinoza (2011), Cázares (2000), Silver y Cai (2005), entre otros, para analizar las producciones a partir de una tarea de invención de problemas aritméticos.

Otro aspecto a resaltar, es que los docentes pueden utilizar las experiencias de los estudiantes para proponer actividades de este tipo, conectando los contenidos matemáticos a enseñar con situaciones de la realidad. Lamentablemente, las actividades de planteamiento de problemas no son una práctica común en las clases de matemática, a pesar de las bondades explícitas en este estudio. Más bien, siguen predominando las actividades de resolución de problemas.

Esto puede ocurrir porque a los docentes se les dificulta emplearlas en sus clases o porque ellos mismos no poseen las competencias necesarias para hacerlo (Leung y Silver, 1997). Además, falta un marco teórico más sólido que pueda ser utilizado al momento de implementar dichas actividades en clases de matemática.

Así, se evidencia que la invención de problemas ha sido objeto de investigación con diferentes propósitos, los cuales aportan resultados valiosos, tanto en la mejora de la educación matemática, como en la aportación de elementos teóricos y metodológicos. De igual forma, es claro que en este tipo de actividades, además de abrir una ventana a través de la cual mirar la comprensión matemática de los estudiantes, también revelan mucho sobre la comprensión de los conocimientos que tienen los estudiantes, sus habilidades, creatividad, significado de los conceptos aprendidos, así como las características de sus experiencias matemáticas escolares.

\section{Agradecimientos}

Agradecemos la colaboración del Proyecto EDU2012-33030 “Procesos de aprendizaje del profesor de matemáticas en formación", del Ministerio de Economía y Competitividad, para llevar a cabo esta investigación. De igual forma, agradecemos la colaboración de la Junta de Becas de la Universidad Nacional de Costa Rica y la Comisión de Incentivos del Consejo Nacional para Investigaciones Científicas y Tecnológicas (CONICIT) del Ministerio de Ciencia y Tecnología de Costa Rica, para llevar a cabo esta investigación.

\section{Bibliografía}

[1] Akay, H. \& Boz, N. (2010). The Effect of Problem Posing Oriented Analyses-II Course on the Attitudes toward Mathematics and Mathematics Self-Efficacy of Elementary Prospective Mathematics Teachers. Australian Journal of Teacher Education, 35(1), 59-75.

[2] AyllónṂ. (2012). Invención-Resolución de problemas por alumnos de educación primaria. Tesis Doctoral. Granada: Universidad de Granada.

[3] Bransford, J. \& Stein, B. (1986). Solución ideal de problemas. Barcelona: Editorial labor

[4] Brown, S. \& Walter, M. (1990). The Art of problem posing. New Jersey: Lawrence Erlbaum Associates.

[5] Brown, S. \& Walter, M. (1993). Problem posing. New Jersey: Lawrence Erlbaum Associates. 
[6] Castro, E. (1991). Resolución de problemas aritméticos de comparación multiplicativa. Memoria de Tercer Ciclo. Departamento de Didáctica de la Matemática. Universidad de Granada.

[7] Castro, E. (2008). Resolución de problemas. Ideas, tendencias e influencias en España. En R. Luengo, B. Gómez, M. Camacho y L. Blanco (Eds.), Investigación en Educación Matemática XII. Actas del Duodécimo Simposio de la Sociedad Española de Investigación en Educación Matemática (pp. 113140). Badajoz: Sociedad Extremeña de Educación Matemática "Ventura Reyes Prósper" y Sociedad Española de Investigación en Educación Matemática.

[8] Cázares, J. (2000). La invención de problemas en escolares de primaria: un estudio evolutivo. Memoria de tercer ciclo. Universidad de Granada.

[9] Cunningham, R. (2004). Problem posing: An opportunity for increasing student responsability. Mathematics and Computer Education, 38(1), 83-89.

[10] Ellerton N. (1986). Children's made up mathematics problems- A new perspective on talented mathematicians. Educational Studies in Mathematics, 17(3), 261-271.

[11] English, L. (1997). The development of fifth-grade children's problem-posing abilities. Educational Studies in Mathematics, 34(3), 183-217.

[12] Espinoza, J. (2011). Invención de problemas aritméticos por estudiantes con talento matemático: Un estudio exploratorio. Memoria de Tercer Ciclo. Universidad de Granada

[13] Freudenthal, H. (1973). Mathematics as an educational task. Dordrecht: Reidel.

[14] Kesan, C., Kaya, D. \& Güvercin, S. (2010). The Effect of Problem Posing Approach to the Gifted Student's Mathematical Abilities. International Online Journal of Educational Sciences, 2(3), 677687.

[15] Kilpatrick, J. (1987). Problem formulating: Where do good problems come from? En A. Shoenfeld (Ed.), Cognitive science and mathematics education (pp 123-148). New Jersey: Lawrance Erlbaum Associates.

[16] Krutetskii, V. A. (1976).The psychology of mathematical abilities in school children. Chicago: University of Chicago Press.

[17] Kwek, M. L. \& Lye, W. L. (2008). Using problem-posing as an assessment tool. Trabajo presentado en $10^{\circ}$ Asia Pacific conference on Giftedness Singapore, Singapur. Descargado el 01/06/2010, de http://hkage.org.hk/en/events/080714_10th_APCG.htm.

[18] Leung, S. \& Silver, E (1997). The role of task format, mathematics knowledge, and creative thinking on the arithmetic problem posing of prospective elementary school teachers. Mathematics Education Research Journal, 9(1), 5-24.

[19] Lin, P. (2004). Supporting teachers on designing problem-posing tasks as a tool of assessment to understand students' mathematical learning. En M. J. Høines y A. B. Fuglestad (Eds.), Proceedings of the 28th Conference of the international. Group for the Psychology of Mathematics Education, Vol. 3 (pp. 257-264). Bergen: Bergen University College.

[20] Luque, A. (2004). La invención de problemas en los que intervienen fracciones por estudiantes del tercer curso de secundaria. Memoria de tercer ciclo. Universidad de Granada.

[21] Moses, B., Bjork, E. \& Goldenberg, E. R. (1990). Beyond problem solving: problem posing. En T. J. Cooney y C. R. Hirsch (Eds.), Teaching and Learning Mathematics in the 1990s (pp. 83-91). Reston, VA: National Council of Teachers of Mathematics.

[22] National Council of Teachers of Mathematics. (1980). An Agenda for Action: Recommendations for School Mathematics of the 1980s. Reston, VA: Autor.

[23] National Council of Teachers of Mathematics. (1989). Curriculum and Evaluation Standards for School of Mathematics. Reston, VA: Autor.

[24] National Council of Teachers of Mathematics. (1991). Professional standards for teaching mathematics. Reston, VA: Autor. 
[25] National Council of Teachers of Mathematics. (2000). Principles and Standards for School Mathematics. Reston, VA: Autor.

[26] OECD (2013). PISA 2012 Assessment and analytical framework. Mathematics, Reading, Science, Problem Solving and Financial Literacy. OECD Publishing. http://dx.doi.org/10.1787/ 9789264190511-en

[27] Polya, G. (1979). Cómo plantear y resolver problemas. México: Trillas.

[28] Pelczer, I. \& Gamboa F. (2008). Problem posing strategies of mathematically gifted students. En R. Leikin (Ed.), Proceedings of the 5th International Conference on Creativity in Mathematics and the Education of Gifted Students (pp 193-199). Tel Aviv: Center for Educational Technology.

[29] Silver, E. A. (1994). On Mathematical Problem Posing. For the Learning of Mathematics, 14(1), 19-28.

[30] Silver, E. \& Cai, J. (1996). An analysis of arithmetic problem posing by middle school students. Journal for Research in Mathematics Education, 27(5), 521-539.

[31] Silver, E. (1997). Fostering creativity through instruction rich in mathematical problem solving and problema posing. ZDM- Zentrallblatt fur Didaktik der Mathematik, 29(3), 75-80.

[32] Silver, E. A. \& Cai, J. (2005). Assessing students' mathematical problem posing. Teaching Children Mathematics, 12(3), 129-135.

[33] Silver, E., Mamona-Downs, J., Leung, S. y Kenney, P (1996). Posing mathematical problem: An exploratory study. Journal for research in matehematics education. 27(3), 293-309.

[34] Stoyanova, E. (1998). Problem posing in mathematics classrooms. En A. McIntosh y N. Ellerton (Eds.), Research in Mathematics Education: a contemporary perspective. (pp 164-185). Edit Cowan University: MASTEC.

[35] Sullivan, P., Clarke, D. \& Clarke, B. (2012). Teaching with tasks for effective mathematics learning. Dordrecht: Springer.

[36] Walter, M. \& Brown, S. (1977): Problem posing and problem solving: an illustration of their interdependence. Matkematics Teacher, 70(1), 5-13. 